\title{
Nudge: Better Quantified-Self with Context-Aware and Proactive Services
}

\author{
Li Guo \\ School of Physical Science and Computing, \\ The University of Central Lancashire \\ lguo@uclan.ac.uk
}

\begin{abstract}
- the concept of quantified-self has drawn great attention along with fast developments of smartphones and wearable sensor technologies. Much work has been focused on life data collection and visualization to help with better self-understanding. However, we argue that although (selfawareness/knowledge discovery is an important aspect of quantified-self, knowledge maintenance is more, or at least equally, important. In this paper, we propose a proactive approach that uses the knowledge mined from people's activity data to nudge them towards a good lifestyle. The trial study is focused on good sleep maintenance. We first use smartphone as an activity detector to collect various features in a non-intrusive manner. We then use those data to learn various activity patterns, induding bedding time, wakeup time and sleep duration. Finally, we analyse correlations that may lead to sufficient or insufficient sleeps and provide customised advices through using proactive services at the right time in order to give them better chances to be turned into actions.
\end{abstract}

\section{Keywords- Quantified-self, Sleep Detection, Activity Learning, Data Mining}

\section{INTRODUCTION}

Along with the fast development of smartphone and wearable sensor technologies, people now are able to collect and gain access to many sources of data about their daily activities and lifestyle patterns. As a consequence, an interesting concept-quantified-self (QS) has been proposed and intensively developed in the last few years. QS is defined as any individual engaged in the selftracking of any kind of personal activity/lifestyle information and/or their associated context in formation. New knowledge about individuals is revealed from the collected data and people are then able to reflect upon it. Nowadays, a wide range of tools or devices for such purposes are available in the market such as Fitbit pedometers [1], Jawbone UP fitness trackers [2] and etc. Also, almost all the solution providers pair their devices or mobile apps with web portals for data aggregation, statistical visualisation. In addition, there are some proposals and work aiming on fusing data from different aspects (activities, exerc ises, work calendar, sleep pattern, food intake, vital signs and etc.) of our life and performing correlations amongst those data. Such work could be very helpful on revealing some hidden knowledge that people may not be aware of. Although some of the existing work has shown their interestingness to be good starting points, making QS to be adopted by a wider public group still faces few main challenges. First of all, the current QS adopters are mainly people who already have strong desire to improve their lifestyles or try to reduce some of the healthy issues that they are experiencing such as poor sleep. Secondly, even the QS technologies can provide more intuitive solutions for knowledge discovery and such knowledge can be understood properly, how people could take further actions in order to benefit from those remains as an issue. In other words, even if people are clearly aware of all potential problems they are having or will have; there is still a big question mark on whether they are "ABLE" to do what they are "SUPPOSED" to. There are many life constraints which stop them from engaging with activities that have proven to be helpful for good lifestyles. Last but not least, almost all of the current QS solutions work on a statistical basis. QS pioneers, more or less, have to have the technical skills that are required to carry the QS tasks (using wearable devices; self-interpreting statistical results aggregated from their data; looking at the charts and understand their meanings and etc.).

To address the above challenges, methods that help to increase people's awareness of importance of QS; help people initiatively engage with QS technologies and constantly take necessary actions in a long run (Knowledge Maintenance) should be studied and deployed. In this paper, we present a set of smart contextaware services that uses the knowledge mined from people's activity data to help them maintain a good lifestyle. This work is specifically targeted at the group of people who have many life constraints. For demonstration purpose, the trial study is focused on good sleep maintenance as it is well known that bad sleep is associated with many potential diseases and has high impact on people' life quality. We first use smartphone as an activity tracker to collect various features unobstructively. We then use those features to learn people's sleep patterns, including bedding/wakeup time and the sleep quality. Finally, we analyse causes that may lead to sufficient or insufficient sleeps and provide customised suggestions at particular time point in order to make them having higher chance to be put into actions. 


\section{RELATED WORK}

\section{A. Quantified-Self, Personal Informatics and Big Data}

QS is also defined as personal analytics and personal informatics. Li [3] proposed the term personal informatics with a stage-based model that is composed of five stages (preparation, collection, integration, reflection, and action), and identified issues people may have in each stage. In his work, he indicated the barrels for both knowledge discovery and knowledge maintenance. This work shows a clear and useful guidance for people to work with QS in a systematic manner. As stated by Swan [4], one of the crucial conceptual that comes with quantified-self is that with all the user lifestyle data available, future healthcare system will not just is a patient's treatment in a personalized $n=1$ manner. But the patient, really a participant, or simply a person, becomes the nexus of action-taking and empowerment. The individual, now through quantified self-tracking and other low-cost newly-available tools, has the ability to understand his or her own patterns and baseline measures, and obtain early warnings as to when there is variance and what to do about this.

Swan [5] also has listed several challenges and opportunities that QS brings to big data community including data storage, data integration and data analys is. Typically a single integrated sensor platform is not available for monitoring participants, say combining sleeping quality with daily activity data (say, exercise amount, food intake, stress level and etc.) but instead the challenges of time alignment, normalised sampling rates and handling missing or error-some data have to be addressed directly as presented by Roantree [6].

\section{B. Engaging People for Quantified Self}

The impression today is that the QS movement is more interested in collecting numbers and transforming them in beautiful representations and visualizations than improving people daily activities. The related work that targets at how to improve user engagement for QS is especially limited to the best of our knowledge.

Social network based service is the mainstream method that is applied to encourage user engagement. Industry wide, almost all the wearable device providers have their own social network community with hopes that users will compare their activities results with others, thus improving the user engagement $[1,7,2]$. Kamal [8] and his colleagues used social network model to track the health behaviour change and engage users. In their prototype system, users are required to provide various life aspect data including mood, entertainment, food and etc. through a web portal based manual logging system. As discussed earlier, this sort of system requires very strong desire of people to engage and is unlikely to be put into practice in a large scale.

Another method is based on augmented reality (often in forms of gaming). Fitness devices and applications are integrated with games running on smartphones. People have to fin ish particular tasks in real world (e.g. jogging for a mile, walking to some places in the city, 40 push-ups in a minute) to proceed further in the game $[9,10]$. Our view on this sort work is that although they are interesting and may be attractive to some of the users (gamers), the "one-size-fits-all" model should be changed to personalised ones that are adaptive to different individual's needs.

Reminding services are also widely adopted by QS applications. Users can set goals, active level or time thresholds as alarms for triggering the reminding services, again, if they have the intention to do so. Recently, more interesting advances of reminding services have been developed. Sleep as android [11], an android smartphone based sleep detecting app can wake people up from sleep without requiring them to setup the alarm clock beforehand. The how it works is that it detects people's sleeping cycle silently and then use the found pattern to decide when the best time to wake people up is. Although, after 3 weeks testing, we found it is still quite immature, it does present an excellent idea-s mart devices can play a proactive role in people's life rather than a reactive one that we usually see.

\section{PROBLEM ANALYSIS AND DESIGN RATIONLE}

From the current literatures, we can see that most works from the QS domain are still heavily focused on the data collection and knowledge discovery stages. People often struggle with many constraints such as time, social responsibilities and much more, which is likely to continue even after we are fully aware of the potential problems. In addition, researches have clearly shown how often people do not make decisions on rational basis, but on irrational thinking, such as heuristics and rules of thumb. It's clear that simple presentation of data and the awareness of people's own condition are not enough to motivate people to modify their habits.

Therefore, we'd rather take an indirect approach with which people do not need to spend time and energy on understanding themselves (if they don't want to) or consciously change their behaviour and habits, but only need to follow advices that they get and act accordingly. In addition to tracking and presenting people lifestyle data, the goal of our research is to investigate methods that could effectively engage users for a long time and nudge changes towards better lifestyle. The fundamental design rationale behind our work is that we try to make QS applications "effortless" for people to use. In this way, an QS application becomes a proactive service which constantly advises people what to do. However, to make such an idea a reality, three primary questions need to be answered.

What are the contents of advices that people would find useful and is willing to accept? If we think about how human solve problems, the rationale process is in the following order: 1> discover the problem, 2> Understand the problem $3>$ find solutions for the problem and $4>$ take actions based on the solutions. This process is exactly what many QS methods are following: using data tracking to help discover problems; using data visualisation and 
statistical methods to help with better understanding; using social communities or specialists' experience to find solutions and no comprehensive solution for step 4 which is actually the most important step. We argue that general public don't need to be initiatively involved in the first three steps. They only need to know what actions to take and how to proceed as what they care about are the final results (e.g. healthier, happier). Therefore, unlike existing QS knowledge representation methods (charting, statis tical summaries and etc.), our work aims at providing advices which people can take direct actions upon.

When is the best moment to send people advices so those advices could have better chances to be put into actions? Conventional reminding services run on a reactive model with which people have to manually setup alarms in advance; get reminded when the alarms are triggered; and take actions accordingly. With such a model, people's actions would normally align with the contents of reminders as they set those themselves. However the proposed "effortless" service works in a proactive way. It should send people advices at the right time when it believes that people need them and have good chance to follow. Such advices cannot be pre-set manually, as they are based on dynamic contexts around people.

What are the bestpresentations for those advices, which will help engage people better? The presentation for the advices is also a very important factor that affects how people react to the given advices. For presentation, we do not refer it to pop up dialogs, text messages or sound alarms as those mechanisms are designed for attracting attentions from people rather than pushing them into actions. What we are really interested in is how to improve the acceptance of the advices sent. For example, sending users a nice picture with a cup of milk and aloud ringtone to their smartphones only reminds them what should be done. How about motivate them to take a picture of the milk that they are going to drink through some game playing and award this behaviour?

Our research aims at seeking answers for these questions. It's crucial to understand $1>$ what are people doing regularly and at what time? 2> what are potential problems of their life (mainly health related)? 3> what are the interruptible time slots during their daily life? We take a data analytic based approach using sleep quality control as an illustration example. Since as a starting point, it's easier to collect feature data about this activity and sleeping, for most of people, shows better patterns than other life activities such as mood. While we were carrying the study, all the methods, system designs and prototyping followed the "effortless" principle. For the proof of concept purposes, we conduct a small-scale deployment using 15 subjects (Age groups ranging from 20 to 40.10 of them are university students and the rests are professionals) for a month for data collection. An android app was developed and loaded into the smartphone used by each subject. The app continuously collects and records the entire feature data. All the subjects are only advised to use their phone regularly without being asked for significant behaviour Collected data are synchronised onto our cloud service [12, 13] continuously every 30 minutes. The system was designed in this way because we intended to carry out all data analysis tasks on the server side.

\section{UNDERST ANDING THE SLEEPING PATTERNS}

To provide useful advices at the right time, we first need to understand users' sleeping patterns including bedding time, wake up time, sleep during and activities that users perform before sleep. To identify different sleep contexts, we've chosen to use the following features (20 features, see Table 1) from a subset of the data collected from our android app.

\begin{tabular}{|l|l|}
\hline Modality & Feature Variables \\
\hline Movement & (Min, Avg, Max, Std) \\
\hline Noise Level & (Min, Avg, Max, Std) \\
\hline Lightness Level & (Min, Avg, Max, Std) \\
\hline Screen on/Off periods & (Min, Avg, Max, Std) \\
\hline User Locations & (Latitude, Longitude) \\
\hline Sleep Time & (Bedding Time, Wake time) \\
\hline
\end{tabular}

Table 1: Selected Features for Sleep Detection

The above table shows the best features that we have experimented with. An interesting finding was the use of user location as a feature, which, as far as we are aware, has not been reported in others' work. As 10 of our subjects are university students, they show very inconsistent sleeping patterns during the period of this study, which led to poor classification performance. One of the significant issues is that they slept in different places (working on a group project late; Friday clubbing; visiting families during weekends are the root causes). Once the places where they sleep changed, the whole contexts followed such changes dramatically. This is especially true for features such as lightness level, movement as well as noise level. Based on such an observation, during the data collection stage, we tagged all the feature data with user locations and later on, during the training process, we trained classification models only using data from the same locations (in our experiments, places in radius within 100 metres range are regarded as a same place). Then, the model that has the same location tags with the time series data is chosen for new classifications. With our approach for each subject, there exist several classification models with different parameter values. Also, the later evaluation results were based the average of overall performance of all models for each individual. To train the classification models, we divided them (from 18:00pm last day to $18: 00 \mathrm{pm}$ current day) into non-overlapped 10 minutes windows. The window size is determined through experiments using different classification models. We tried 4 different window sizes ( 10 minutes, 15 minutes, 20 minutes and 30 minutes). The 10 minutes window performed the best over the other sizes on all the models that were tried. For a new classification, after all the windows are classified, we merge them into bigger chunks in order to gain feedbacks from the participants.

We tried several classification models in order to achieve the best performance. To train those models, we used 25 days data and performed some data pre-processing tasks. 
This ends up with 3505 10-minutes window instances for each subject. To evaluate those models, 10-fold crossvalidation was applied. From the results, we can conclude that none of single feature gave acceptable results apart from the "sleeping time" feature which has to be manually supplied by the participants. However, while putting them together, the performance boosts dramatically. The best result is achieved via using Random Forest, which shows an average of $95.48 \%$ accuracy on classifying sleeping windows with F-value at 0.91.

\section{NUDGING THROUGH PROACTIVE SERVICES}

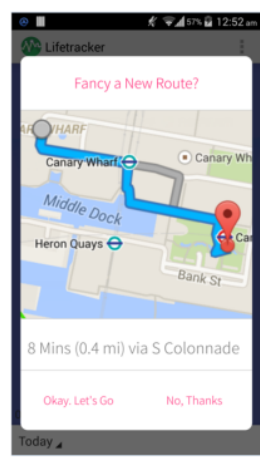

a

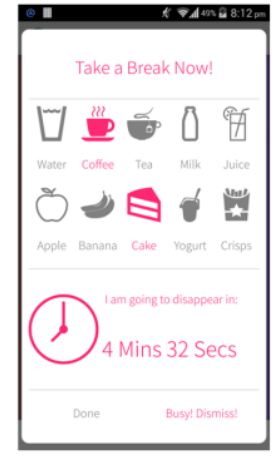

b

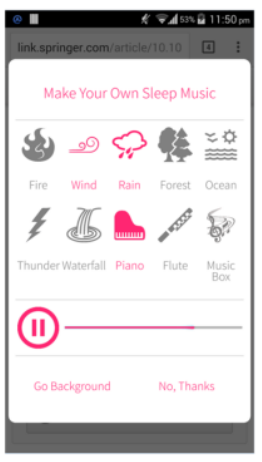

C
Figure 1: Nudging Services for Encouraging More steps, Less App Use Time and Relaxing before Bedding

Based on the analysis results from the previous section, we are now able to generate customised nudging advices for each individual. As discussed earlier, the key challenge is how to engage users better when they receive those advices and how we can possibly evaluate whether the advices are accepted by the users without explicitly asking for feedbacks. Our focus is to advise people what to do "right now". We designed three advising mechanis ms aiming at improving daily amount of steps; reducing continuous screen time/app usages and making people relax before/during their sleep.

A. Nudging for More Steps

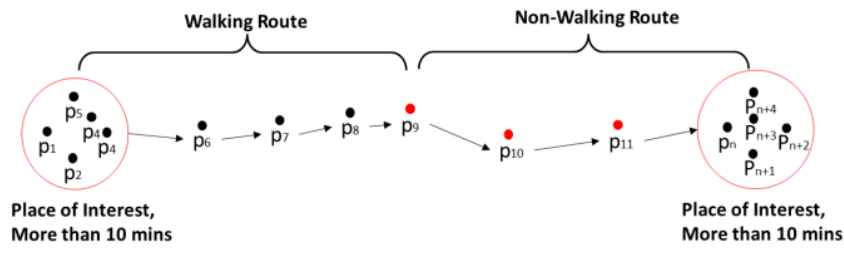

Figure 2: Walking Routes Detection

The main approach that we designed to nudging for more steps is to remind users using alternative paths for their reoccurring trajectories, say from home to tube station, from tube station to work and etc. To automatically detect the reoccurring trajectories we first need to identify all their walking routes on a single day. Figure 2 shows the basic idea.

For each day (except Saturday and Sunday as we found walking routes on these two days show large variations), we first sort all the user's location data between his/her last wakeup time and the current bedding time. Then we cluster all location points that are connected sequentially and are within 10 metres distance. For each cluster, we calculate their centrals (using KMeans); label the centrals as points of interest and use them as "break points" to separate all location data from that day into several segments. At the last, for each segment, we filter out the consecutive data points that have higher distances than a pre-set threshold (for our work, we use 150 metres as our data collection frequency is 1 minute).

To learn reoccurring trajectories, we used all working routes from two weeks and compare the similarities between them iteratively. For a pair of routes that needs to be compared, Google places service is integrated to obtain street name for each of the location points (house numbers and postcodes are removed and same street names for one route are discarded). The result street names are then concatenated into a larger string in the same order as the location points for each walking route. Finally, we calculate the edit distance between the two larger strings and if the value is less than a pre-set threshold, the two walking routes are regarded as a same trajectory.

All the reoccurring trajectories found are tagged with their starting location, end location, starting clock time, end clock time, average steps taken in between, average walking speed, map distance (obtained from Google navigation service) as well as all street names. Given these information, alternative routes (using Google Map API) that connect the same start location and end location for a reoccurring trajectory are retrieved and stored locally. The alternative route that costs more steps will be advised to the user. We didn't use the route that has the largest step counts as it has higher chance to be rejected. If no alternative routes are found or an alternative route has been continuously rejected for more than 3 times, no advice will be generated for that trajectory anymore.

To evaluate the performance of the above proposed approach, we carried out our experiments over 20 days. The first 10 days were used to calculate reoccurring trajectories and the last 10 days were used to generate advices. In the experiment, we tested how many advices were seen by users and were accepted in the last 10 days against the total reoccurring trajectories that actually took place in the first 10 days. All participants were randomly divided into two groups of equal size of 10 . In the first group users' app, the alternative route advices were generated using our proposed approach while for the second group of users, advices were generated randomly before a reoccurring trajectory takes place on a day.

The results are shown in Figure 3, from which we can see that with our approach (Figure 3.a) for group one, 63.19\% of alternative advices were seen by users (an advice is only considered as "seen" if it has an user feedback recorded) with acceptance rate at $41.73 \%$ (whether a user has accepted an advice or not was measured by comparing his walking route with the suggested route after the advices were provided). For group two ((Figure 3.b), although similar amount of advices were generated seen by users $(53.68 \%)$, the acceptance ratio is much lower at $19.09 \%$. This confirms that even we know users 
activities/behaviours, when to remind them about behaviour changes will affect the results significantly.

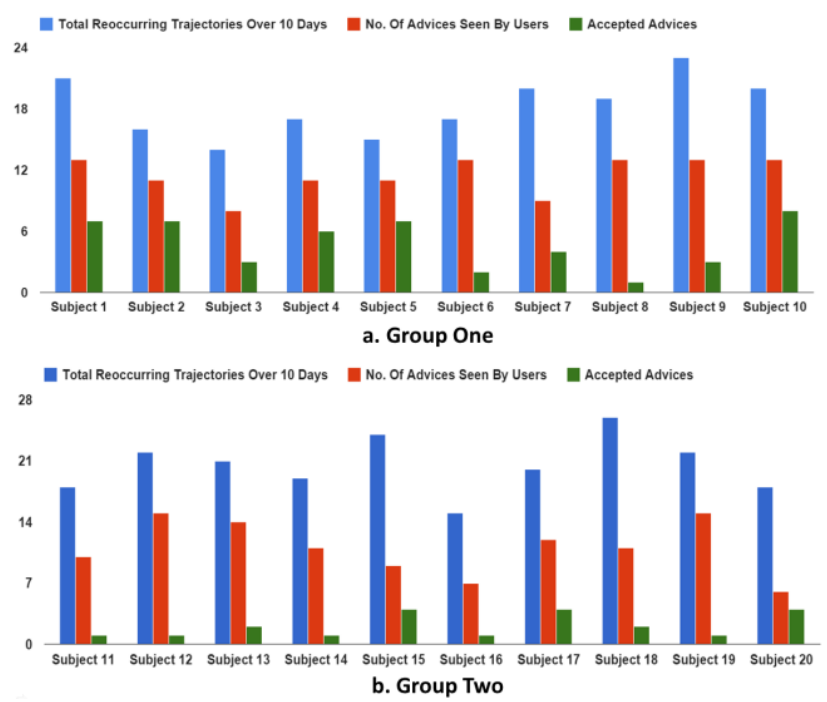

Figure 3: Total Reoccurring Trajectories VS No. of Alternative Route Advices Generated VS No. Of Accepted Advices.

There is also an average $3.03 \%$ steps increasing for the first group users in 10 days as shown in Figure 4. This amount doesn't seem to be significant at first glance. However, with our approach, if users keep accepting advices in a long run, this number will keep going up as the new routes that we advise always have higher step counts than the current one. The trend will continue till it coverages at some points (say, the longest path is taking by users, or users choose to use a shorter path constantly).

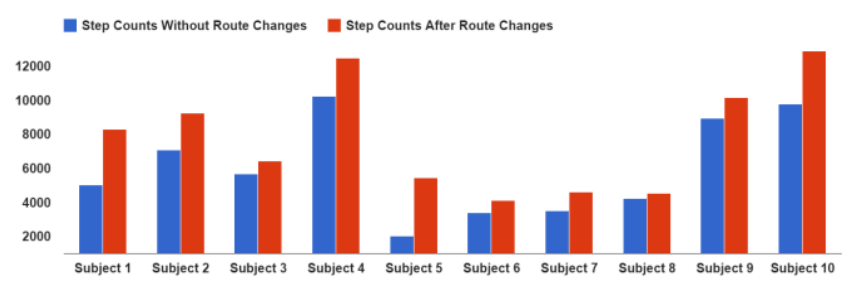

Figure 4: Step Counts Changes (without Route Change VS with Route Changes)

\section{B. Nudging For Less App Time Usage}

Although addiction to mobile devices or apps is a wellknown problem, most of the existing works mainly target at kids' mobile usage control. Very limited effort work can be found for adults to the best of our knowledge. Using password to stop people using their mobile phones or pre-set a limited use time cannot effectively reduce the mobile use time for those who have less initiative. Apps that are designed this way normally end up with being uninstalled as they are very likely to disturb the normal phone usages. Instead, our work for tackling this problem focused on gentle disturbances. As shown in Figure 1.b, we developed a service that sends advices to users to remind them for breaks. The service doesn't explicitly ask users to stop using their phones/apps, but only suggests breaks of snacks or drinks. Moreover, it doesn't need to be pre-set for a regular repeating time or for particular apps. It learns when is the best time to send those advices, which is modelled as another classification problem. Once a user accepts the break reminder, he can also log what he has done during the break. Although the logged data such as water intake, calories are not used for this work, they are reserved for feature studies. Random forest is adopted as the classification model using 16 features. Total 732 instances were collected in 10 days (sleeping hours were filtered out) from each subject (group of 10) for training. 10-folds cross validation was applied for evaluating the model. The result shows an average acceptance accuracy at $63.23 \%$ (Prec ision: 0.576, Recall: 0.610, F-Value: 0.59, RMSE: 90.23\%).

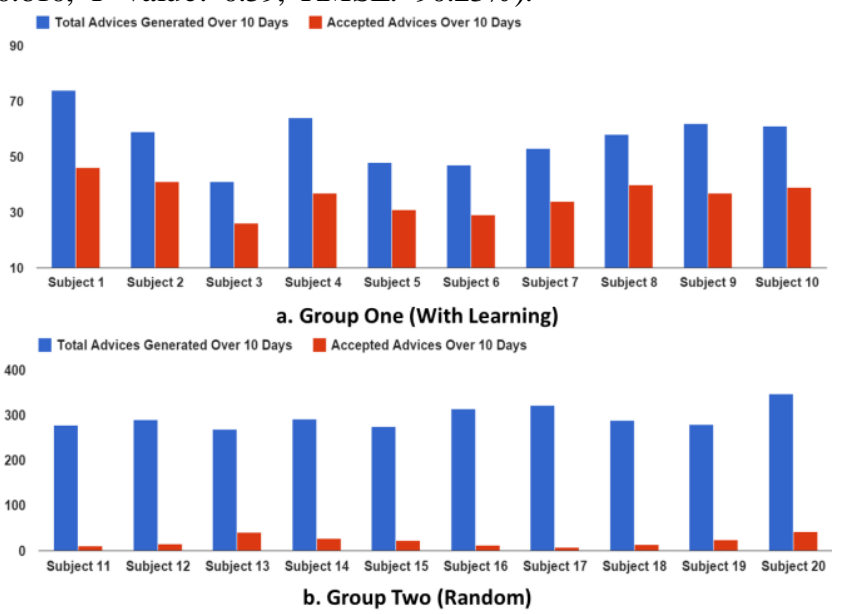

Figure 5: App Disturbance Advice Acceptance Rate (Leaning VS Random)

We have to admit this result doesn't look very promising on its own. However, while being applied in later testing stages (another 10 days for testing), it outperformed the results from random advices generation (advices are generated with irregular intervals between 30 minutes and an hour) in terms of the number of accepted advices (See Figure 5, random advice generation only received $7.3 \%$ acceptances). In addition, total app usages reduced $10.03 \%$ averagely across the 10 subjects in group one with our proposed approach as shown in Figure 6.

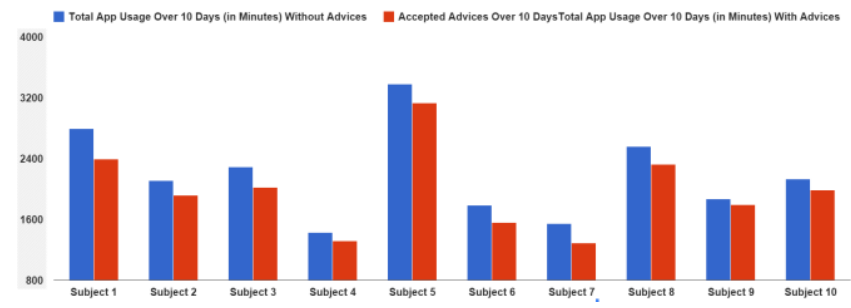

Figure 6: Total App Use Time Reduction

In addition, we noticed that, from the experiments, there are no clear corre lations between the nu mber of generated advices, the number of accepted advices and the app use time reduction. Further experiments need to be carried out to see the rules behind.

\section{Nudging For Earlier Bedding Time}

The last service that we designed aims at relaxing people before they go to sleep with hopping that getting them relaxed earlier could make them go to bed earlier and 
sleep better. The service is designed as a simple game using which people can combine different sound tracks/effects into a melody that they feel comfortable with. Sound effects can be added or removed one by one in real time so users are able to hear the change immediately without stopping or restarting the track. The service is ready to be triggered an hour before the "best" bedding time that's learnt from the correlation study for each individual. Similar to the alternative route service, a pop up reminder (see Figure 1.c) appears at the time when a user is using his phone after the reminder is ready. If "go background" is clicked, the sound track keeps playing until it's stopped by users manually or by the service if it detects that the user is asleep. Also, using the accelerometer and lightness data, the service adjusts the sound volume down little by little. Moreover, the service automatically $\log s$ information including the combination of sound effects for every track play; length of the sound tracks play; start time of play; end time of play; how is a play terminated (by useror by service).

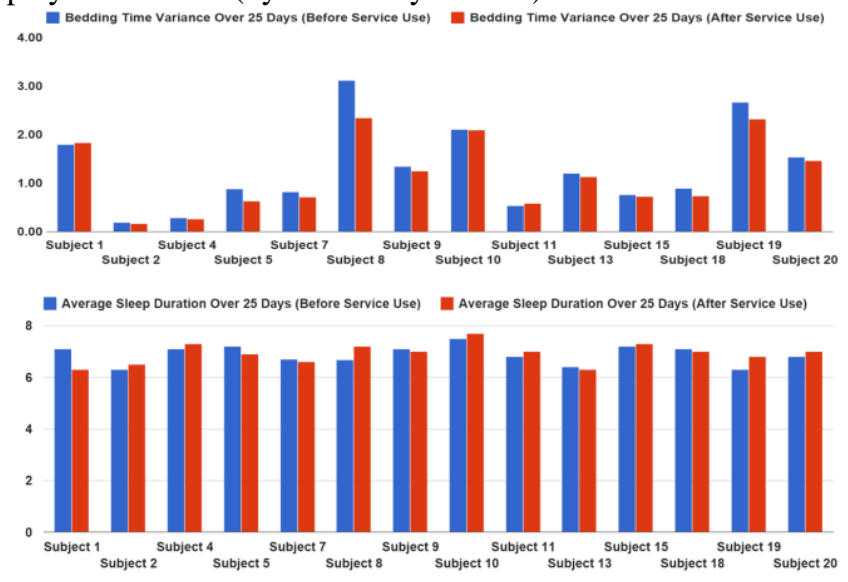

Figure 7: Bedding Time Variance and Average Sleep Duration Changes (before Service Use VS after Service Use)

The experiment for this service ran for another 25 days right after the sleep detection data collection periods. Data of participants who had not used the service regularly (less than 10 minutes averagely for each use) are removed from the study. Figure 7 shows the change of bedding time variances before and after service use, from which we can see for people who had regularly bedding times, using this service doesn't change the regularity much. However, for the two extreme cases (subject 8 \& subject $19)$, the bedding time variances did reduce to a noticeable level, so are the incensements for their sleep durations.

\section{CONCLUSION}

In this paper, we proposed a "nudging" based approach to help people achieve better lifestyles with less effort. We believe the work presented in this paper has the following contributions to the big data, personal informatics and healthcare communities:

$1>$ The concept of using proactive services for quantified-self applications in order to minimise people's effort.
2> A set of proactive services which learn user behaviour automatically and use learnt knowledge to nudge peoples' activities without requiring too much manual intervention.

In our future work, we plan to incorporate more features into the existing study. Data for many other features have been collected such as water amount, calories intake amount and etc. We also plan to design methods and services that learn people's mood/stress level through unobtrusive interactions.

\section{REFERENCES}

[1] FitBit.http://www.fitbit.com.

[2] Jawbone.https://jawbone.com/.

[3] I. Li, A. K. Dey and J. Forlizzi, "Understanding My Data, Myself: Supporting Self-reflection with Ubicomp Technologies," in Proceedings of the 13th International Conference on Ubiquitous Computing, New York, NY, USA, 2011.

[4] M. Swan, "Health 2050: the realization of personalized medicine through crowdsourcing, the Quantified Self, and the participatory biocitizen," Journal of Personalized Medicine, vol. 2, no. 3, pp. 93-118, 2012.

[5] M. Swan, "The quantified self: Fundamental disruption in big data science and biological discovery," Big Data, vol. 1, no. 2, pp. 85-99, 2013.

[6] M. Roantree, J. Shi, P. Cappellari, M. F. O'Connor, M. Whelan and N. Moyna, "Data trans formation and query management in personal health sensor networks," Journal of Network and Computer Applications, vol. 35, no. 4, pp. 1191-1202, 2012.

[7] Nike+.https://secure-nikeplus.nike.com/plus/.

[8] N. Kamal, S. Fels and K. Ho, "Online Social Networks for Personal Informatics to Promote Positive Health Behavior," in Proceedings of Second ACM SIGMM Workshop on Social Media, New York, NY, USA, 2010.

[9] BallStrike.http://www.fit-master.com/.

[10] ZombiesRun. https://www.zombiesrungame.com/.

[11] Sleep as Android. https://sites.google.com/site/sleepasandroid/.

[12] Y. Li, L. Guo, C. Wu, C.-H. Lee and Y. Guo, "Building a cloud-based platform for personal health sensor data management," in Biomedical and Health Informatics (BHI), 2014 IEEE-EMBS International Conference on, 2014.

[13] Y. Li, C. Wu, L. Guo, C.-H. Lee and Y. Guo, "WikiHealth: A Big Data Platform for Health," Cloud Computing Applications for Quality Health Care Delivery, p. 59, 2014. 